\title{
THE LIGHT CURVE OF SN 1987A REVISITED: CONSTRAINING PRODUCTION MASSES OF RADIOACTIVE NUCLIDES
}

\author{
Ivo R. SeitenZahl ${ }^{1,2,3}$, F. X. Timmes ${ }^{4,5}$, and Georgios MagKotsios ${ }^{5}$ \\ ${ }^{1}$ Research School of Astronomy and Astrophysics, Mount Stromlo Observatory, Cotter Road, Weston Creek, ACT 2611, Australia; ivo.seitenzahl@anu.edu.au \\ ${ }^{2}$ Universität Würzburg, Emil-Fischer-Straße 31, D-97074 Würzburg, Germany \\ ${ }^{3}$ Max-Planck-Institut für Astrophysik, Karl-Schwarzschild-Straße 1, D-85748 Garching, Germany \\ ${ }^{4}$ School of Earth and Space Exploration, Arizona State University, Tempe, AZ 85287, USA \\ ${ }^{5}$ The Joint Institute for Nuclear Astrophysics, Notre Dame, IN 46556, USA \\ Received 2012 October 13; accepted 2014 June 27; published 2014 August 8
}

\begin{abstract}
We revisit the evidence for the contribution of the long-lived radioactive nuclides ${ }^{44} \mathrm{Ti},{ }^{55} \mathrm{Fe},{ }^{56} \mathrm{Co},{ }^{57} \mathrm{Co}$, and ${ }^{60} \mathrm{Co}$ to the UVOIR light curve of SN 1987A. We show that the $V$-band luminosity constitutes a roughly constant fraction of the bolometric luminosity between 900 and 1900 days, and we obtain an approximate bolometric light curve out to 4334 days by scaling the late time $V$-band data by a constant factor where no bolometric light curve data is available. Considering the five most relevant decay chains starting at ${ }^{44} \mathrm{Ti},{ }^{55} \mathrm{Co},{ }^{56} \mathrm{Ni},{ }^{57} \mathrm{Ni}$, and ${ }^{60} \mathrm{Co}$, we perform a least squares fit to the constructed composite bolometric light curve. For the nickel isotopes, we obtain best fit values of $M\left({ }^{56} \mathrm{Ni}\right)=(7.1 \pm 0.3) \times 10^{-2} M_{\odot}$ and $M\left({ }^{57} \mathrm{Ni}\right)=(4.1 \pm 1.8) \times 10^{-3} M_{\odot}$. Our best fit ${ }^{44} \mathrm{Ti}$ mass is $M\left({ }^{44} \mathrm{Ti}\right)=(0.55 \pm 0.17) \times 10^{-4} M_{\odot}$, which is in disagreement with the much higher $(3.1 \pm 0.8) \times 10^{-4} M_{\odot}$ recently derived from INTEGRAL observations. The associated uncertainties far exceed the best fit values for ${ }^{55} \mathrm{Co}$ and ${ }^{60} \mathrm{Co}$ and, as a result, we only give upper limits on the production masses of $M\left({ }^{55} \mathrm{Co}\right)<7.2 \times 10^{-3} M_{\odot}$ and $M\left({ }^{60} \mathrm{Co}\right)<1.7 \times 10^{-4} M_{\odot}$. Furthermore, we find that the leptonic channels in the decay of ${ }^{57} \mathrm{Co}$ (internal conversion and Auger electrons) are a significant contribution and constitute up to $15.5 \%$ of the total luminosity. Consideration of the kinetic energy of these electrons is essential in lowering our best fit nickel isotope production ratio to $\left[{ }^{57} \mathrm{Ni} /{ }^{56} \mathrm{Ni}\right]=2.5 \pm 1.1$, which is still somewhat high but is in agreement with gamma-ray observations and model predictions.
\end{abstract}

Key words: nuclear reactions, nucleosynthesis, abundances - supernovae: general supernovae: individual (SN 1987A)

Online-only material: color figures

\section{INTRODUCTION}

The set of radioactive parent isotopes that have been used to model the nuclear decay energy source terms for the light curve of SN 1987A are ${ }^{22} \mathrm{Na},{ }^{44} \mathrm{Ti},{ }^{56} \mathrm{Ni},{ }^{57} \mathrm{Ni}$, and ${ }^{60} \mathrm{Co}$ (e.g., Pinto et al. 1988; Woosley et al. 1989; Timmes et al. 1996). It is commonly held that ${ }^{44} \mathrm{Ti}$ decay plays a dominant role beyond 2000 days (e.g., Lundqvist et al. 2001; Fransson \& Kozma 2002; Motizuki \& Kumagai 2004; Jerkstrand et al. 2011; Larsson et al. 2011). Recently, it was shown that heating by internal conversion and Auger electrons emitted during the decay of ${ }^{57} \mathrm{Co}$ and Auger electrons produced in the decay of ${ }^{55} \mathrm{Fe}$ can be the dominant channels for the light curves of thermonuclear supernovae (Seitenzahl et al. 2009; Seitenzahl 2011; Röpke et al. 2012). In this paper, we re-evaluate the light curve of SN 1987A, taking into account these previously neglected decay channels.

Several observational and theoretical efforts have estimated the mass of radioactive ${ }^{44} \mathrm{Ti}$ synthesized in SN 1987A-see Table 1 for a compilation of results and references. Most of the derived observational estimates and model predictions do not agree within their respective uncertainties. For example, modeling the strengths of metal emission lines in the nebular phase leads to ${ }^{44} \mathrm{Ti}$ masses $\gtrsim 1.0^{-4} M_{\odot}$, while upper limits derived from space-based infrared spectroscopy generally find few $\times 10^{-5} M_{\odot}$ of ${ }^{44} \mathrm{Ti}$. Analysis of $400 \mathrm{ks}$ of Chandra ACIS data suggests a $2 \sigma$ upper limit of $M\left({ }^{44} \mathrm{Ti}\right)<2 \times 10^{-4} M_{\odot}$ (Leising 2006), while analysis of 6 Ms of hard X-ray data taken with the IBIS/ISGRI instrument on INTEGRAL suggests $(3.1 \pm 0.8) \times$
$10^{-4} M_{\odot}$ of ${ }^{44} \mathrm{Ti}$ (Grebenev et al. 2012). Spherically symmetric hydrodynamic models of SN 1987A progenitors tend to produce few $\times 10^{-5} M_{\odot}$ of ${ }^{44} \mathrm{Ti}$, explosions models with high energies and artificially imparted asymmetries in two dimensions appear to produce few $\times 10^{-4} M_{\odot}$ of ${ }^{44} \mathrm{Ti}$ along the poles of the model explosions (e.g., Nagataki et al. 1997, 1998), and efforts to model the SN 1987A light curve cluster around $M\left({ }^{44} \mathrm{Ti}\right) \approx$ $1.0^{-4} M_{\odot}$. At face value, these model predictions for the mass of ${ }^{44} \mathrm{Ti}$ ejected are smaller than allowed by the uncertainties of the INTEGRAL measurement (Grebenev et al. 2012).

Since ${ }^{56} \mathrm{Ni}$ and ${ }^{57} \mathrm{Ni}$ are short lived, their mass ratio $M\left({ }^{57} \mathrm{Ni}\right) /$ $M\left({ }^{56} \mathrm{Ni}\right)$ is often expressed in units of the corresponding ratio of the final decay products in the Sun, i.e., $\left[{ }^{57} \mathrm{Ni} /{ }^{56} \mathrm{Ni}\right]=$ $\left[M\left({ }^{57} \mathrm{Ni}\right) / M\left({ }^{56} \mathrm{Ni}\right)\right] /\left[M\left({ }^{57} \mathrm{Fe}\right) / M\left({ }^{56} \mathrm{Fe}\right)\right] \odot$. The solar ratio is $\left[M\left({ }^{57} \mathrm{Fe}\right) / M\left({ }^{56} \mathrm{Fe}\right)\right]_{\odot} \approx 0.0235$ (Cameron 1982; Anders \& Grevesse 1989; Rosman \& Taylor 1998; Lodders 2003; Asplund et al. 2009). The mass ratio of ${ }^{57} \mathrm{Ni}$ to ${ }^{56} \mathrm{Ni}$ in SN 1987A, which is prominently affecting light curve models between 900-1800 days, has not reached consensus between the values inferred from observations and light curve models (see Table 1). For example, $\left[{ }^{57} \mathrm{Ni} /{ }^{56} \mathrm{Ni}\right]=1.5 \pm 0.3^{\text {stat }} \pm 0.2^{\text {sys }}$ was derived from the gamma ray flux of ${ }^{57} \mathrm{Co}$ with the OSSE instrument on the Compton Observatory (Kurfess et al. 1992), upper limits of $M\left({ }^{57} \mathrm{Co}\right)<2.8 \times 10^{-3} M_{\odot}$ from the HEXE instrument aboard MIR-KVANT also correspond to roughly ratios of 1.5 (Sunyaev et al. 1991), and interpretations of ground-based infrared spectroscopy also favor similar ratios. Such moderate enhancement ratios are also in agreement with spherically symmetric and asymmetric hydrodynamic models 
Table 1

Measured, Inferred, and Predicted Yield Values for SN 1987A

\begin{tabular}{|c|c|c|c|c|c|}
\hline $\begin{array}{l}M\left({ }^{55} \mathrm{Co}\right)^{\mathrm{a}} \\
{\left[10^{-3} M_{\odot}\right]}\end{array}$ & $\begin{array}{c}M\left({ }^{60} \mathrm{Co}\right) \\
{\left[10^{-4} M_{\odot}\right]}\end{array}$ & {$\left[{ }^{57} \mathrm{Ni} /{ }^{56} \mathrm{Ni}\right]$} & $\begin{array}{c}M\left({ }^{44} \mathrm{Ti}\right) \\
{\left[10^{-4} M_{\odot}\right]}\end{array}$ & Method & References \\
\hline$\cdots$ & $\cdots$ & $\cdots$ & $3.1 \pm 0.8$ & Soft gamma-ray (IBIS/ISGRI, INTEGRAL) & 1 \\
\hline$\lesssim 1$ & $\cdots$ & $\cdots$ & $<2.0(2 \sigma)$ & X-ray (ACIS, Chandra) & 2 \\
\hline$\cdots$ & $\cdots$ & $<1.5$ & $<90$ & X-ray (HEXE/Mir-Kvant) & 3 \\
\hline$\cdots$ & $\cdots$ & $1.5 \pm 0.3^{\text {stat }} \pm 0.2^{\mathrm{sys}}$ & $\cdots$ & Gamma-ray (OSSE/Compton GRO) & 4 \\
\hline$\cdots$ & $\cdots$ & $1-2$ & $\cdots$ & Infrared spectroscopy (FIGS/AAT) & 5 \\
\hline$\cdots$ & $\cdots$ & $\lesssim 1.5$ & $\cdots$ & Infrared spectroscopy (ESO) & 6 \\
\hline$\cdots$ & $\cdots$ & $\cdots$ & $<0.15$ & Infrared spectroscopy (ISO/SWS) & 7 \\
\hline$\cdots$ & $\cdots$ & $\cdots$ & $<0.59^{\mathrm{b}}$ & Infrared spectroscopy (ISO/SWS) & 8 \\
\hline$\cdots$ & $\cdots$ & $\cdots$ & 4.00 & Nebular emission lines $(H S T)$ & 9 \\
\hline$\cdots$ & $\cdots$ & $\cdots$ & $1.0-2.0$ & Nebular emission lines (HST) & 10 \\
\hline$\cdots$ & $\cdots$ & $\cdots$ & $1.4 \pm 0.5$ & Nebular emission lines $(H S T)$ & 11 \\
\hline$\cdots$ & $\cdots$ & $\gtrsim 5$ & 0.92 & UVOIR light curve & 12 \\
\hline$\cdots$ & $\cdots$ & $4.5 \pm 1.6$ & 1.00 & UVOIR light curve & 13 \\
\hline$\ldots$ & $\cdots$ & $5 \pm 1$ & 1.00 & UVOIR light curve & 14 \\
\hline$\ldots$ & $\ldots$ & $\sim 2$ & 1.00 & UVOIR light curve & 15 \\
\hline$\cdots$ & $\cdots$ & $\sim 2$ & $0.5-2.0$ & UVOIR light curve & 16 \\
\hline$\ldots$ & $\cdots$ & $0.5-2.5$ & $\lesssim 0.85$ & Pure nuclear reaction network calculation & 17 \\
\hline$\cdots$ & $\cdots$ & $\sim 1.0$ & $\lesssim 0.3$ & Explosion model/network calculations & 18 \\
\hline 0.3 & $3.6 e-10$ & 1.7 & 1.7 & Explosion model/network calculations & 19 \\
\hline 1.3 & 1.6 & 1.3 & 0.26 & S19 explosion model/network calculations & 20 \\
\hline$\ldots$ & $\ldots$ & $0.5-2.0$ & $\lesssim 0.5$ & Explosion model/network calculations & 21 \\
\hline$\cdots$ & $\ldots$ & $\ldots$ & $\int_{0.5}$ & Explosion model/network calculations & 22 \\
\hline$<7.2$ & $<1.7$ & $2.5 \pm 1.1$ & $0.5 \tilde{5} \pm 0.17$ & Least squares light curve fitting & This work \\
\hline
\end{tabular}

Notes.

a This is actually the combined mass of ${ }^{55} \mathrm{Co}$ and ${ }^{55} \mathrm{Fe}$, but since most of the mass in the $A=55$ decay chain is synthesized as ${ }^{55} \mathrm{Co}$ and $\tau\left({ }^{55} \mathrm{Co}\right) \ll \tau\left({ }^{55} \mathrm{Fe}\right)$ (see Section 3.1), we write the sum of the masses as $M\left({ }^{55} \mathrm{Co}\right)$.

${ }^{\mathrm{b}}$ Extreme assumptions about clumping and positron escape increase this upper limit to $<1.1$.

References. (1) Grebenev et al. 2012; (2) Leising 2006; (3) Sunyaev et al. 1991; (4) Kurfess et al. 1992; (5) Varani et al. 1990; (6) Bouchet \& Danziger 1993; (7) Borkowski et al. 1997; (8) Lundqvist et al. 2001; (9) Wang et al. 1996; (10) Chugai et al. 1997; (11) Jerkstrand et al. 2011; (12) Kumagai et al. 1991; (13) Dwek et al. 1992; (14) Suntzeff et al. 1992; (15) Fransson \& Kozma 1993; (16) Fransson \& Kozma 2002; (17) Woosley \& Hoffman 1991; (18) Woosley \& Weaver 1995; (19) Thielemann et al. 1996; (20) Rauscher et al. 2002; (21) Nomoto et al. 2006; (22) Tur et al. 2010.

of SN 1987A progenitors, which produce $\left[{ }^{57} \mathrm{Ni} /{ }^{56} \mathrm{Ni}\right]$ between 0.5 and 2.5. On the other hand, values of $\left[{ }^{57} \mathrm{Ni} /{ }^{56} \mathrm{Ni}\right] \approx 5$ times solar were initially derived from light curve modeling (Kumagai et al. 1991; Suntzeff et al. 1992; Dwek et al. 1992). Such an enhanced ratio was challenged by theoretical nucleosynthesis considerations (Woosley \& Hoffman 1991), which limit $\left[{ }^{57} \mathrm{Ni} /{ }^{56} \mathrm{Ni}\right]$ to at most four times solar and place the most likely value between 0.5 and 2.5 times solar (see also Section 2). Subsequently, time-dependent models that allow for the effects of non-equilibrium ionization have been introduced as a solution to the overproduction problem. Based on such calculations, values for $\left[{ }^{57} \mathrm{Ni} /{ }^{56} \mathrm{Ni}\right]$ as low as two times solar have been claimed to be in agreement with the observations (Fransson \& Kozma 1993, 2002), although we maintain that the four times solar $\left[{ }^{57} \mathrm{Ni} /{ }^{56} \mathrm{Ni}\right]$ case in Figure 3 of Fransson \& Kozma (1993) actually provides a much better fit to the data.

Seitenzahl (2011) estimated that including the usually ignored contribution of Auger and internal conversion electrons of ${ }^{57} \mathrm{Co}$ and ${ }^{55} \mathrm{Fe}$ in the heating budget might make significant contributions to the light curve of SN 1987A. In this paper, we refine that estimate. In Section 2, we discuss constraints on $\left[{ }^{57} \mathrm{Ni} /{ }^{56} \mathrm{Ni}\right]$ from standard, parameterized postexplosion freeze-out profiles. In Section 3, we present our analytic light curve model and discuss extending the observed UVOIR pseudo-bolometric light curves of SN 1987A to later times; in Section 4, we present the results of modeling the extended light curve. We find that (1) the $(3.1 \pm 0.8) \times 10^{-4} M_{\odot}$ of ${ }^{44} \mathrm{Ti}$ derived by Grebenev et al. (2012) from gamma-ray observations with INTEGRAL are in conflict with our much lower value of $M\left({ }^{44} \mathrm{Ti}\right)=(0.55 \pm 0.17) \times 10^{-4} M_{\odot}$ required to explain the luminosity of the late light curve; (2) including the internal conversion and Auger electrons produced in ${ }^{57} \mathrm{Co}$ decay reduces the mass of ${ }^{57} \mathrm{Ni}$ required for light curve models; (3) within our uncertainties, ${ }^{60} \mathrm{Co}$ may be the dominant source of radioactive energy injection for a few years at intermediate times between. In Section 5, we conclude with a summary.

\section{CONSTRAINING $\left[{ }^{57} \mathrm{Ni} /{ }^{56} \mathrm{Ni}\right]$ FROM PARAMETERIZED FREEZE-OUT PROFILES}

Figure 1 shows $\left[{ }^{57} \mathrm{Ni} /{ }^{56} \mathrm{Ni}\right]$ produced from post-explosion freeze-out expansion calculations for exponential thermodynamic trajectories within a large grid of peak temperatures and peak densities (Magkotsios et al. 2010, 2011). Both ${ }^{56} \mathrm{Ni}$ and ${ }^{57} \mathrm{Ni}$ belong in the second family of isotopes that are produced during freeze-out expansions (see Table 2 of Magkotsios et al. 2011). Isotopes of the second family become nuclear flow hubs, dominate the final composition, and do not sustain any transition between equilibrium states during the evolution. This implies relatively featureless contour plots of final yields compared to the isotopes of the first family which do undergo a transition between equilibrium states during the evolution. The structure of $\left[{ }^{57} \mathrm{Ni} /{ }^{56} \mathrm{Ni}\right]$ in Figure 1 stems from a varying, relative efficiency between ${ }^{56} \mathrm{Ni}$ and ${ }^{57} \mathrm{Ni}$ in absorbing nuclear flows. Overall, the 


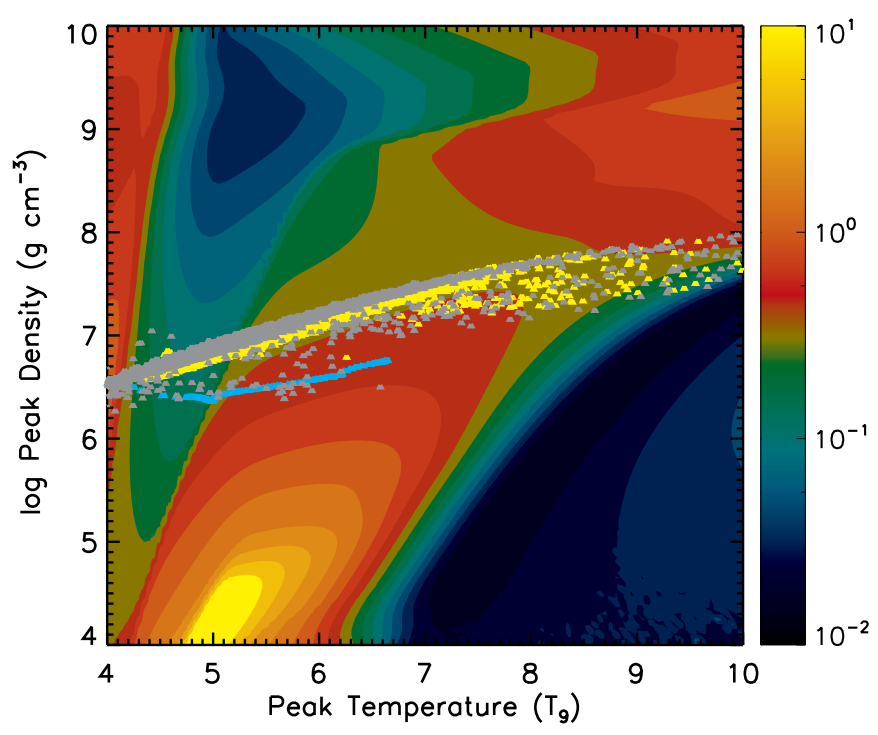

Figure 1. Final $\left[{ }^{57} \mathrm{Ni} /{ }^{56} \mathrm{Ni}\right.$ ] after freeze-out from exponential thermodynamic trajectories for an electron fraction of $Y_{e}=0.5$ as a function of peak temperature and peak density (Magkotsios et al. 2010, 2011). Overplotted are the peak thermodynamic conditions reached in three multi-dimensional corecollapse supernova simulations of SN 1987A (cyan: P. Young \& C. Fryer 2012, private communication; yellow: A. Wongwathanarat \& T. Janka 2012, private communication; gray: Wongwathanarat et al. 2010).

(A color version of this figure is available in the online journal.)

doubly magic nucleus ${ }^{56} \mathrm{Ni}$ is the most efficient flow hub among the isotopes of the second family near the magic number 28 . The relative strength of ${ }^{57} \mathrm{Ni}$ to ${ }^{56} \mathrm{Ni}$ in absorbing flow has a weak dependence on the type of freeze-out. In particular, ${ }^{57} \mathrm{Ni}$ is not very efficient for the $\alpha p$-rich freeze-out region and parts of the $\mathrm{Si}$-rich and normal freeze-out regions. For the photodisintegration regime at the bottom right part of Figure 1 neither isotope is produced.

Figure 1 also shows the peak conditions taken from three multi-dimensional supernova simulations of SN 1987A to constrain the accessible $\left[{ }^{57} \mathrm{Ni} /{ }^{56} \mathrm{Ni}\right]$. Figure 1 suggests that in these simulations most of the parameterized trajectories produce ratios near or below unity. The total $\left[{ }^{57} \mathrm{Ni} /{ }^{56} \mathrm{Ni}\right]$ ratio of all mass elements from the exponential trajectories are generally are within a factor of $\sim 2$ of the ratios found by post-processing the multidimensional SN 1987A supernova simulations with the same 489 isotope reaction network used for the parameterized trajectories. These results are consistent with measurements in the 1.5-2 range listed in Table 1.

\section{THE UVOIR LIGHT CURVE}

We base our analysis on the UVOIR pseudo-bolometric light curves (i.e., not counting escaping gamma-rays) instead of a wavelength dependent radiative transfer calculation. Figure 2 shows $V$-band data to 4334 days (Leibundgut \& Suntzeff 2003; Fransson et al. 2007) and UVOIR data to 1854 days (Suntzeff et al. 1992; Suntzeff 1997) for SN 1987A. Owing to their long half-lives, the radionuclides ${ }^{44} \mathrm{Ti},{ }^{55} \mathrm{Fe}$, and ${ }^{60} \mathrm{Co}$ mainly affect the bolometric light curve in the later phases after $\sim 1200$ days. To constrain the mass ejected by these isotopes, we notice the $V$-band is an approximately constant fraction of the bolometric light between 840 and 1854 days, as shown by the inset of Figure 2. We construct an approximate bolometric light curve for longer than 1854 days by scaling the $V$-band by a constant factor of 12.6. This provides an additional ten UVOIR data

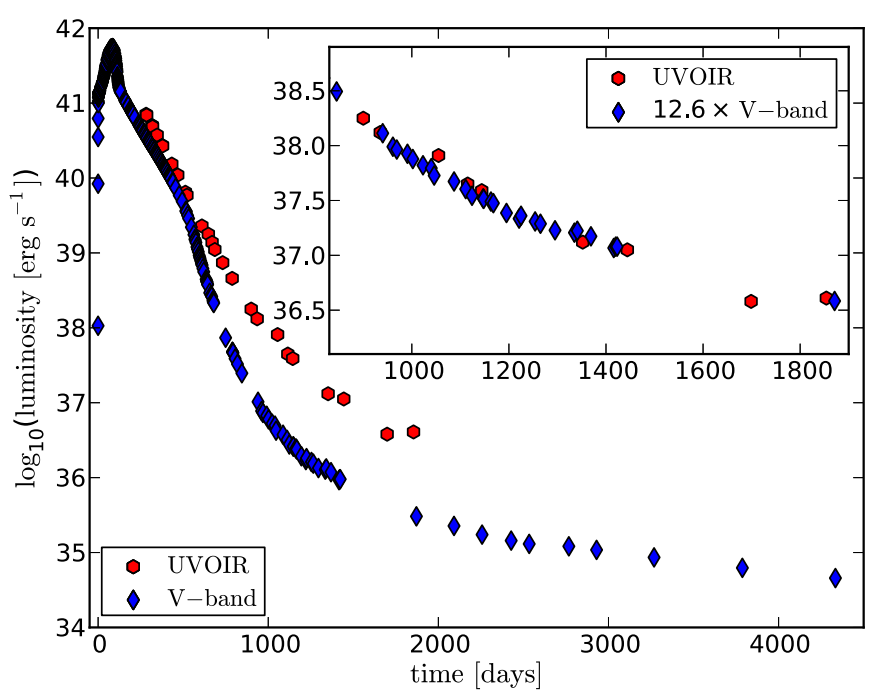

Figure 2. UVOIR (Suntzeff et al. 1992; Suntzeff 1997) and V-band (Leibundgut \& Suntzeff 2003; Fransson et al. 2007) light curves for SN 1987A. The inset shows that the $V$-band is approximately a constant factor of 12.6 times the bolometric light curve between 840 and 1854 days. We use this agreement to approximate the UVOIR light from the $V$-band by assuming the same scaling behavior holds also between days 1854 and 4436 .

(A color version of this figure is available in the online journal.)

points at late times that correspond to the ten measured $V$-band data points shown in Figure 2.

\subsection{Light Curve Model}

We model the bolometric light curve with effective opacities following Woosley et al. (1989) and Timmes et al. (1996). We assume that only radioactive energy input contributes to the luminosity and approximately accounts for energy stored in the ionization of the ejecta and released at a later time. We include energy deposited by Auger and internal conversion electrons, and we use up to date nuclear decay information from the National Nuclear Data Center ${ }^{6}$ (see Table 2). We consider contributions from the following five decay chains to the bolometric supernova light curve:

$$
\begin{aligned}
& { }^{56} \mathrm{Ni} \stackrel{t_{1 / 2}=6.08 d}{\longrightarrow}{ }^{56} \mathrm{Co} \stackrel{t_{1 / 2}=77.2 d}{\longrightarrow}{ }^{56} \mathrm{Fe} \\
& { }^{57} \mathrm{Ni} \stackrel{t_{1 / 2}=35.60 h}{\longrightarrow}{ }^{57} \mathrm{Co} \stackrel{t_{1 / 2}=271.79 d}{\longrightarrow}{ }^{57} \mathrm{Fe} \\
& { }^{55} \mathrm{Co} \stackrel{t_{1 / 2}=17.53 h}{\longrightarrow}{ }^{55} \mathrm{Fe} \stackrel{t_{1 / 2}=999.67 d}{\longrightarrow}{ }^{55} \mathrm{Mn} \\
& { }^{44} \mathrm{Ti} \stackrel{t_{1 / 2}=58.9 y}{\longrightarrow}{ }^{44} \mathrm{Sc} \stackrel{t_{1 / 2}=3.97 h}{\longrightarrow}{ }^{44} \mathrm{Ca} \\
& { }^{60} \mathrm{Co} \stackrel{t_{1 / 2}=5.27 y}{\longrightarrow}{ }^{60} \mathrm{Ni} .
\end{aligned}
$$

We do not consider the ${ }^{22} \mathrm{Na}$ decay chain since nucleosynthesis calculations show the ${ }^{55} \mathrm{Co}$ chain always injects significantly more energy than the ${ }^{22} \mathrm{Na}$ chain, and ${ }^{55} \mathrm{Fe}$ and ${ }^{22} \mathrm{Na}$ have very similar half-lives of $\sim 2.7$ and $\sim 2.6 \mathrm{yr}$, respectively.

\footnotetext{
6 http://www.nndc.bnl.gov/
} 
Table 2

Charged Lepton $\left(q^{l}\right)$ and Gamma-Ray $\left(q^{\gamma}\right)$ Partial Radioactive Decay Energies Per Decay, Effective Opacities $(\kappa)$, and Decay Constants $(\lambda)$

\begin{tabular}{lcccc}
\hline \hline Nucleus & $\begin{array}{c}\lambda \\
{\left[d^{-1}\right]}\end{array}$ & $\begin{array}{c}q^{l} \\
{[\mathrm{keV}]}\end{array}$ & $\begin{array}{c}q^{\gamma} \\
{[\mathrm{keV}]}\end{array}$ & $\begin{array}{c}\kappa \\
{\left[\mathrm{cm}^{2} \mathrm{~g}^{-1}\right]}\end{array}$ \\
\hline${ }^{60} \mathrm{Co}$ & $3.600 \mathrm{e}-4$ & 96.41 & 2504 & $0.04^{\mathrm{a}}$ \\
${ }^{57} \mathrm{Co}$ & $2.551 \mathrm{e}-3$ & 17.82 & 121.6 & 0.0792 \\
${ }^{56} \mathrm{Co}$ & $8.975 \mathrm{e}-3$ & 119.4 & 3606 & 0.033 \\
${ }^{55} \mathrm{Fe}$ & $6.916 \mathrm{e}-4$ & 3.973 & $1.635^{\mathrm{b}}$ & $\ldots$ \\
${ }^{44} \mathrm{Ti}$ & $3.222 \mathrm{e}-5$ & 596.0 & 2275 & 0.04 \\
\hline
\end{tabular}

Notes.

${ }^{\text {a }}$ We use the same value as Timmes et al. (1996).

b Counting X-rays.

The time-dependence of $n$ nuclide abundances $N_{i}$ in a decay chain is governed by the Bateman equations:

$$
\begin{aligned}
& \frac{d N_{1}}{d t}=-\lambda_{1} N_{1} \\
& \frac{d N_{i}}{d t}=\lambda_{i-1} N_{i-1}-\lambda_{i} N_{i} .
\end{aligned}
$$

For $n=2$ and initial abundances $N_{1}(0)$ and $N_{2}(0)$, we get the solution

$$
\begin{aligned}
N_{1}(t)= & N_{1}(0) \exp \left(-\lambda_{1} t\right) \\
N_{2}(t)= & N_{1}(0) \frac{\lambda_{1}}{\lambda_{2}-\lambda_{1}}\left[\exp \left(-\lambda_{1} t\right)-\exp \left(-\lambda_{2} t\right)\right] \\
& +N_{2}(0) \exp \left(-\lambda_{2} t\right)
\end{aligned}
$$

The decay constants $\lambda_{i}$ are related to the half-lives $t_{1 / 2, i}$ and the mean lifetime $\tau_{i}$ via

$$
\lambda_{i}=\frac{1}{\tau_{i}}=\frac{\ln (2)}{t_{1 / 2, i}} .
$$

The rate of energy deposition by decays of nucleus $i$ is given by the activity multiplied by the energy deposited per decay:

$$
\epsilon_{i}=\lambda_{i} N_{i}(t) q_{i}(t)
$$

where the number $N_{i}$ is given by Equations (8) or (9) and the energy deposited, $q_{i}$, is a function of time due to the increasing escape fraction of gamma-rays and possible late time escape of positrons.

To reduce the number of variables, we make use of the large difference in half-lives in four of the decay chains:

$$
\begin{gathered}
\tau\left({ }^{44} \mathrm{Ti}\right) \gg \tau\left({ }^{44} \mathrm{Sc}\right) \\
\tau\left({ }^{55} \mathrm{Co}\right) \ll \tau\left({ }^{55} \mathrm{Fe}\right) \\
\tau\left({ }^{56} \mathrm{Ni}\right) \ll \tau\left({ }^{56} \mathrm{Co}\right) \\
\tau\left({ }^{57} \mathrm{Ni}\right) \ll \tau\left({ }^{57} \mathrm{Co}\right) .
\end{gathered}
$$

This allows us to approximate the solution to the Bateman equations with a single exponential for each decay chain. We refer to the luminosity in the $A=44$ chain, where $A$ is the atomic number, as being due to the long-lived ${ }^{44} \mathrm{Ti}$, even though the positron is actually produced in the subsequent decay of ${ }^{44} \mathrm{Sc}$. Using effective opacities for the gamma-rays and hard $\mathrm{X}$-rays and assuming instantaneous and complete deposition of the leptonic kinetic energy, we obtain the following timedependent expression for the luminosity due to a given decay chain,

$$
L_{A}(t)=2.221 \frac{\lambda_{A}}{A} \frac{M(A)}{M_{\odot}} \frac{q_{A}^{l}+q_{A}^{\gamma} f_{A}}{\mathrm{keV}} \exp \left(-\lambda_{A} t\right) \times 10^{43} \mathrm{erg},
$$

where $q^{l}$ and $q^{\gamma}$ are the average energies per decay carried by charged leptons and gamma-rays, respectively (see Table 2), $f_{A}=\left\{1.0-\exp \left[-\kappa_{A} \phi_{0}\left(t_{0} / t\right)^{2}\right]\right\}, \phi_{0}=7.0 \times 10^{4} \mathrm{~g} \mathrm{~cm}^{-2}$ is the column density at the fiducial time $t_{0}=10^{6} \mathrm{~s}$, and $A$ stands for the atomic numbers $\{44,55,56,57,60\}$ of the five decay chains.

Next, we describe how we take time-dependent freeze-out effects into account (see Fransson \& Kozma 1993, 2002). Freeze-out is most significant in the hydrogen envelope. As recombination times lengthen, the relative importance of freezeout effects initially increases, as long as gamma-rays still deposit significant energy in the envelope. The ${ }^{57} \mathrm{Co}$ and ${ }^{60} \mathrm{Co}$ electrons, as well as the ${ }^{44} \mathrm{Sc}$ positrons, are produced deep in the core and have very short mean free paths. Complete and instantaneous thermalization is therefore still a good approximation for these charged particle contributions. Therefore, upon entering the positron-/electron-dominated phase, recombination times in the hydrogen envelope become more or less irrelevant, which leads to the disappearance of time-dependent recombination effects. This behavior is clearly shown in Figure 2 of Fransson \& Kozma (2002), in which time-dependent effects begin to make a difference starting at $\sim 1000$ days and increase in importance until $\sim 1600$ days. From then on, the difference between the time-dependent and the steady state calculations decreases steadily until $\sim 2800$ days when the time-dependent and the steady-state results converge again.

We extract the freeze-out contribution $L_{\text {freeze }}(t)$ with a plot digitizer from Figure 1 of Fransson \& Kozma (1993) by taking the difference between the two curves labeled " ${ }^{56} \mathrm{Ni}$-only" and extrapolating after 2000 days. We assume a linear dependence of the freeze-out correction on the initial ${ }^{56} \mathrm{Ni}$ mass, i.e., the term that is added to the bolometric luminosity is $\left(M\left({ }^{56} \mathrm{Ni}\right) / 0.07 M_{\odot}\right) L_{\text {freeze }}(t)$. Such a freeze-out correction scaling linearly with $M\left({ }^{56} \mathrm{Ni}\right)$ appears to be a good approximation since addition of, e.g., two times solar ${ }^{57}$ Co does not significantly affect the freeze-out contribution (see Figure 1 of Fransson \& Kozma 1993). In other words, most of the freeze-out luminosity is energy stored in ionization from the ${ }^{56} \mathrm{Co}$-dominated phase, which justifies our approach.

\section{CONFRONTING THE LIGHT CURVE DATA WITH OBSERVATIONS AND THEORY}

In the previous section, we constructed a bolometric light curve of SN 1987A from published bolometric data points where available (out to 1854 days) and scaled $V$-band light at later times. In the following, we use this composite light curve to constrain production masses of radionuclides.

We begin with a comparison of our constructed UVOIR light curve with the predictions of our light curve model for canonical production masses of the radionuclides ${ }^{56} \mathrm{Ni},{ }^{57} \mathrm{Ni}$, and ${ }^{44} \mathrm{Ti}$. Figure 3 shows the model UVOIR light curve and the luminosity of the decay chains using canonical masses of 


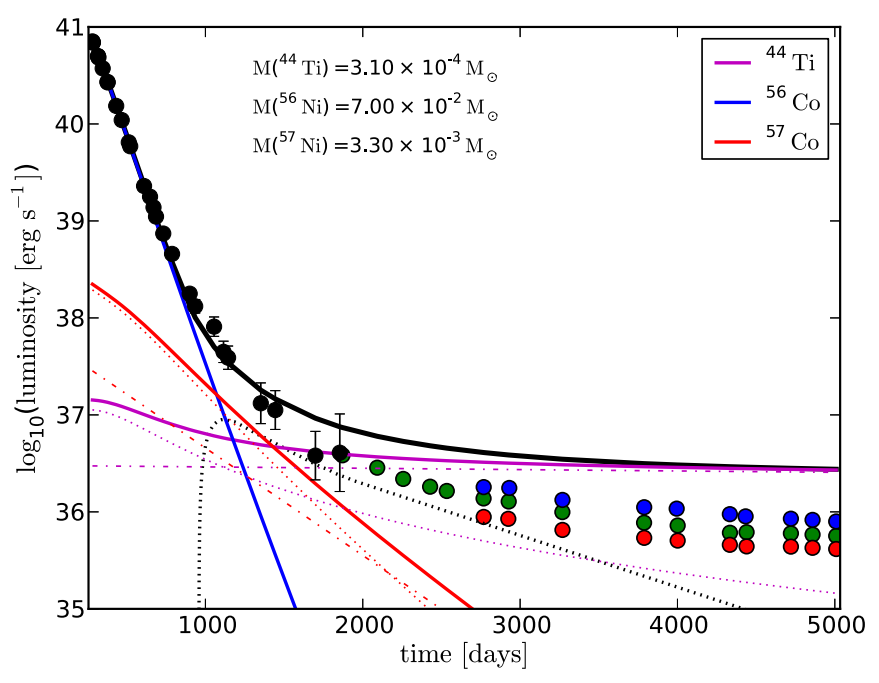

Figure 3. Model light curve for canonically accepted values of ${ }^{56} \mathrm{Ni}$ and ${ }^{57} \mathrm{Ni}$ (Fransson \& Kozma 1993) and the ${ }^{44} \mathrm{Ti}$ value of Grebenev et al. (2012). The partial $\gamma$-ray contributions for each nuclide are shown with dotted lines and electron and positron contributions are shown with dot-dashed lines. The solid black line is the model bolometric UVOIR luminosity when the energy stored in ionization and released later through recombination is approximately taken into account. The dotted black line shows this freeze-out term, i.e., our model for the delayed release of energy stored in ionization. In black are published UVOIR data for SN 1987A (Suntzeff et al. 1992; Suntzeff 1997). In green are scaled $V$-band data (Leibundgut \& Suntzeff 2003; Fransson et al. 2007). Arbitrarily scaled $B$-band (blue) and $R$-band (red) data extracted from the paper of Larsson et al. (2011) are shown to demonstrate that a similar rate of decline is present in other optical filters.

(A color version of this figure is available in the online journal.)

$M\left({ }^{56} \mathrm{Ni}\right)=7.0 \times 10^{-2} M_{\odot}$ and $M\left({ }^{57} \mathrm{Ni}\right)=3.3 \times 10^{-3} M_{\odot}$ (Fransson \& Kozma 2002) and the recently determined $M\left({ }^{44} \mathrm{Ti}\right)=3.1 \times 10^{-4} M_{\odot}($ Grebenev et al. 2012). Green circles in Figure 3 are the scaled and time extended $V$-band data where the scaling corresponds to a constant fraction of $\sim 8 \%$ (factor 12.6). Figure 3 also shows the arbitrarily scaled $R$-band (red circles) and $B$-band (blue circles) data we extracted from Figure 2 of Larsson et al. (2011). Note that the $B, V$, and $R$ bands evolve quite similarly: there is apparently not much color evolution during these later epochs. The fact that the $R$ and $B$ bands fall off in a similar manner and bracket the $V$ band in wavelength lends credence to our extrapolation of the $V$-band scaling performed in Section 3. Note that all color light curves fall off with a timescale much faster than ${ }^{44} \mathrm{Ti}$ and that $M\left({ }^{44} \mathrm{Ti}\right)=3.1 \times 10^{-4} M_{\odot}$ results in a luminosity at late times significantly exceeding our composite light curve.

Next, we perform a non-linear, non-weighted least-squares fit to the logarithm of the composite light curve constructed in the previous section. We use ScIPy curvefit, which employs the Levenberg-Marquardt algorithm. We fit the data with the light curve model consisting of the three traditionally employed radionuclides ${ }^{56} \mathrm{Ni},{ }^{57} \mathrm{Ni}$, and ${ }^{44} \mathrm{Ti}$, as well as ${ }^{55} \mathrm{Co}$ and ${ }^{60} \mathrm{Co}$ and the freeze-out term (see Section 3.1). The result of the fit is shown in Figure 4. For the nickel isotopes, we obtain best fit values of $M\left({ }^{56} \mathrm{Ni}\right)=(7.1 \pm 0.3) \times 10^{-2} M_{\odot}$ and $M\left({ }^{57} \mathrm{Ni}\right)=(4.1 \pm 1.8) \times 10^{-3} M_{\odot}$. Note that we obtain $M\left({ }^{44} \mathrm{Ti}\right)=(5.5 \pm 1.7) \times 10^{-5} M_{\odot}$, a value much smaller than the INTEGRAL measurement of $(3.1 \pm 0.8) \times 10^{-4} M_{\odot}$ (Grebenev et al. 2012), but very similar to what is obtained from explosion models and nuclear reaction network calculations (see Table 1).

The half-lives of ${ }^{60} \mathrm{Co}$ and ${ }^{55} \mathrm{Fe}$ are quite similar, which introduces a degeneracy for the fitting algorithm. As a result, their best fit values are much smaller than the associated uncertainties.

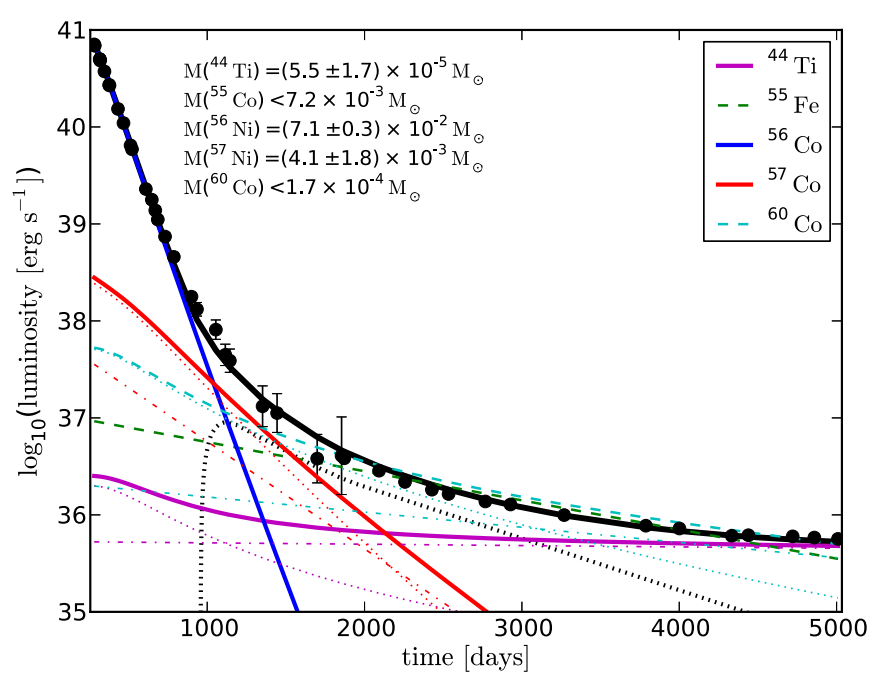

Figure 4. Model light curve (thick black line) including our time-dependent freeze-out correction (black dashed line). This light curve is the result of a fivecomponent least squares fit of the initial abundances of ${ }^{44} \mathrm{Ti},{ }^{56} \mathrm{Ni},{ }^{57} \mathrm{Ni},{ }^{55} \mathrm{Co}$, and ${ }^{60} \mathrm{Co}$ on the composite bolometric light curve constructed in Section 3. The partial $\gamma$-ray contributions for each nuclide are shown with dotted lines and electron and positron contributions are shown with dot-dashed lines. The best fit values of ${ }^{55} \mathrm{Co}$ and ${ }^{60} \mathrm{Co}$ are significantly smaller than their respective uncertainties, which we show as upper limits for these isotopes.

(A color version of this figure is available in the online journal.)

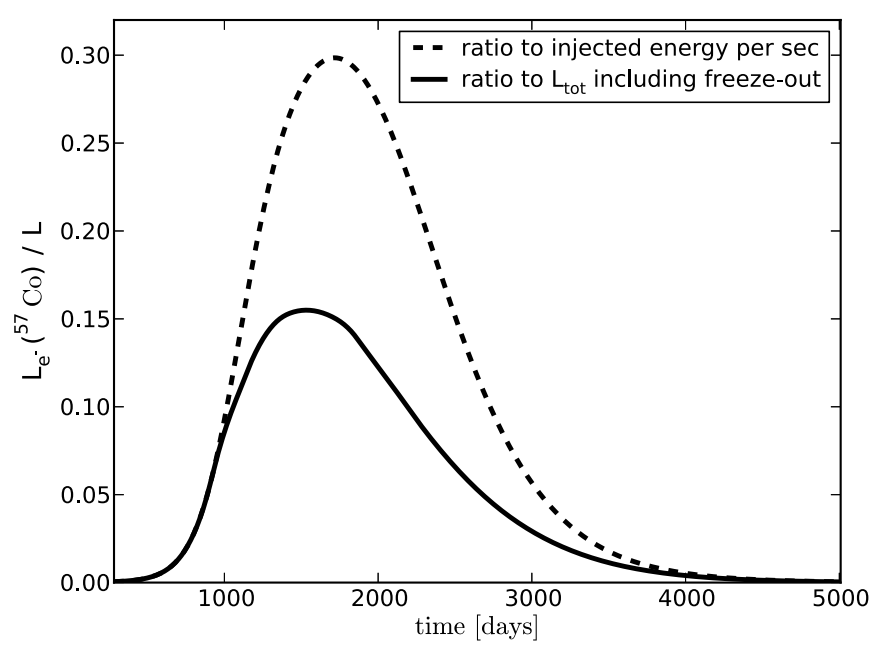

Figure 5. Relative importance of the ${ }^{57} \mathrm{Co}$ electrons for the light curve of SN 1987A. The solid line is the ratio of the energy injection from Auger and internal conversion electrons of ${ }^{57} \mathrm{Co}$ (thin red dot-dashed line in Figure 4) to the total luminosity including freeze-out for our best-fit solution shown in Figure 4 (solid black line). The dashed line is the ratio of the energy injection from Auger and internal conversion electrons of ${ }^{57} \mathrm{Co}$ to the instantaneous total rate of energy injection from radioactive decay (solid black line minus dotted black line in Figure 4).

While we use the best fit values of $M\left({ }^{55} \mathrm{Co}\right)=9.2 \times 10^{-6} M_{\odot}$ and $M\left({ }^{60} \mathrm{Co}\right)=4.5 \times 10^{-8} M_{\odot}$ for our combined light curve in Figure 4, we plot the nuclide specific heating terms for the upper limits on the relevant production masses of $M\left({ }^{55} \mathrm{Co}\right)<$ $7.2 \times 10^{-3} M_{\odot}$ and $M\left({ }^{60} \mathrm{Co}\right)<1.7 \times 10^{-4} M_{\odot}$.

For SN 1987A, the leptonic channels of ${ }^{57} \mathrm{Co}$ (thin red dash-dotted line) play a lesser role compared to the case of thermonuclear supernovae. Nevertheless, their instantaneous, relative contribution to the total bolometric luminosity peaks at 1533 days at $15.5 \%$ and constitutes over $10 \%$ between 1058 and 2189 days (see Figure 5). It is interesting to note that the effect 


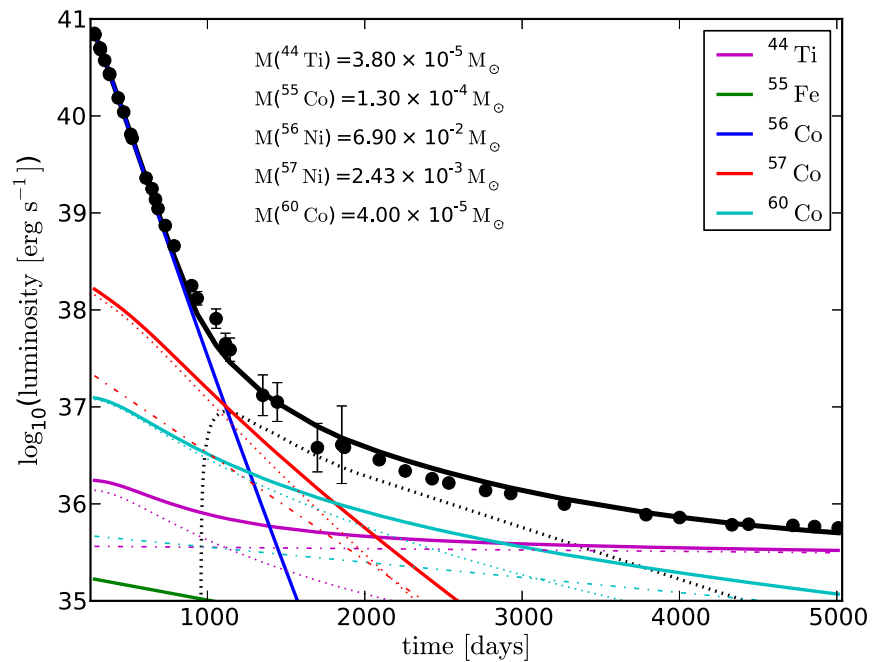

Figure 6. Model light curve (thick black line) including our time-dependent freeze-out correction (dashed black line) for a choice of initial masses that results in a good fit to the data. For ${ }^{44} \mathrm{Ti},{ }^{57} \mathrm{Co}$, and ${ }^{60} \mathrm{Co}$, gamma-ray (thin dotted lines) and leptonic (thin dash-dotted lines) partial light curves are shown separately as in Figures 3 and 4.

(A color version of this figure is available in the online journal.)

of these electron channels on the best fit $\left[{ }^{57} \mathrm{Ni} /{ }^{56} \mathrm{Ni}\right]$ is even larger. If we perform a fit to the light curve with the electron channels of ${ }^{57} \mathrm{Co}$ omitted, we obtain $\left[{ }^{57} \mathrm{Ni} /{ }^{56} \mathrm{Ni}\right]=3.8 \pm 1.0$, whereas our best fit of the light curve that includes the heating from internal conversion and Auger electrons of ${ }^{57} \mathrm{Co}$ yields $\left[{ }^{57} \mathrm{Ni} /{ }^{56} \mathrm{Ni}\right]=2.5 \pm 1.1$.

Figure 6 demonstrates that a qualitatively good match to the observed light curve is possible where ${ }^{60} \mathrm{Co}$ dominates the energy injection in the epoch between the ${ }^{57} \mathrm{Co}$ and ${ }^{44} \mathrm{Ti}$ dominated phases. Such a scenario is a viable possibility, and we advocate that ${ }^{60} \mathrm{Co}$ should be included in all late light curve and spectral models of SN 1987A. Again, we have taken the time-dependent effects of freeze-out in the hydrogen envelope approximately into account. This hypothetical model demonstrates that a good match to the light curve data of SN 1987A can be obtained with radionuclides other than ${ }^{44} \mathrm{Ti}$ (i.e., ${ }^{60} \mathrm{Co}$ ) dominating the heating at intermediate times.

The luminosity of X-rays produced in radioactive decays is generally very small compared to the other radiation from decays (e.g., positrons, electrons, gamma-rays) and neglecting Xrays for the heating is therefore generally a good approximation. This ansatz has been made in all previous works that fitted the light curve of SN 1987A. We note, however, that since the decay of ${ }^{55} \mathrm{Fe}$ is a ground state to ground state transition, no gamma-rays are emitted and X-rays constitute $\sim 29 \%$ of the total liberated decay energy ( $\sim 41 \%$ of the leptonic contribution). The contribution of X-rays is therefore quite significant for this particular nucleus and for simplicity we work in the limit of full trapping and local thermalization of ${ }^{55} \mathrm{Fe} \mathrm{X}$-rays.

\section{SUMMARY}

We have confirmed previous claims (Seitenzahl 2011) that the leptonic heating channels (internal conversion and Auger electrons) from the decay of ${ }^{57} \mathrm{Co}$ are a significant source of energy for the light curve of SN 1987A, constituting as much as $30 \%$ of the instantaneous total rate of energy injection from radioactive decay. For our best-fitting abundances of the radionuclides, the ${ }^{57} \mathrm{Co}$ electrons constitute up to $15.5 \%$ of the total luminosity at 1533 days (see Figure 5). Our best fit of the light curve that includes the heating from internal conversion and Auger electrons of ${ }^{57} \mathrm{Co}$ yields $\left[{ }^{57} \mathrm{Ni} /{ }^{56} \mathrm{Ni}\right]=2.5 \pm 1.1$, down from $\left[{ }^{57} \mathrm{Ni} /{ }^{56} \mathrm{Ni}\right]=3.8 \pm 1.0$ for the case where these channels are omitted.

Here we only give upper limits $M\left({ }^{55} \mathrm{Co}\right)<7.2 \times 10^{-3} M_{\odot}$ and $M\left({ }^{60} \mathrm{Co}\right)<1.7 \times 10^{-4} M_{\odot}$ on the production masses of the cobalt isotopes. Within the uncertainties of our light curve analysis, e.g., a ${ }^{60} \mathrm{Co}$ dominated phase remains a possibility in the epoch between the ${ }^{57} \mathrm{Co}-$ and ${ }^{44} \mathrm{Ti}$-dominated phases. We note, however, that our best fit to the reconstructed bolometric light curve (including the freeze-out term) does not require any significant power from either ${ }^{55} \mathrm{Fe}$ or ${ }^{60} \mathrm{Co}$.

We investigated the impact of the recently determined ${ }^{44} \mathrm{Ti}$ mass of $3.1 \times 10^{-4} M_{\odot}$. We find that published, fully reconstructed data is still reconcilable with this high value, but that the $V, B$, and $R$ bands at late times indicate that a much lower value would match the light curve better. Indeed, our fit of the constructed bolometric light curve gives $M\left({ }^{44} \mathrm{Ti}\right)=(0.55 \pm 0.17) \times 10^{-4} M_{\odot}$, a value in good agreement with most explosion models and nuclear reaction network calculations.

We thank Annop Wongwathanarat, Thomas Janka, Chris Fryer, and Patrick Young for making their core collapse supernova simulation data available to us. We also thank Josefin Larsson, Roland Diehl, and the anonymous referee for their helpful comments. I.R.S. was supported by the ARC Laureate Grant FL0992131, the Deutsche Forschungsgemeinschaft via the Emmy Noether Program (RO 3676/1-1), and GRK-1147. G.M. was supported by the NSF under Grant PHY 08-22648 for the Frontier Center "Joint Institute for Nuclear Astrophysics" (JINA). F.X.T. was partially supported by NSF AST1107484.

\section{REFERENCES}

Anders, E., \& Grevesse, N. 1989, GeCoA, 53, 197

Asplund, M., Grevesse, N., Sauval, A. J., \& Scott, P. 2009, ARA\&A, 47, 481

Borkowski, K. J., de Kool, M., McCray, R., \& Wooden, D. H. 1997, BAAS, 29, 1269

Bouchet, P., \& Danziger, I. J. 1993, A\&A, 273, 451

Cameron, A. G. W. 1982, in Essays in Nuclear Astrophysics, ed. C. A. Barnes, D. D. Clayton, \& D. N. Schramm (Cambridge: Cambridge Univ. Press), 23

Chugai, N. N., Chevalier, R. A., Kirshner, R. P., \& Challis, P. M. 1997, ApJ, 483, 925

Dwek, E., Moseley, S. H., Glaccum, W., et al. 1992, ApJL, 389, L21

Fransson, C., Gilmozzi, R., Groeningsson, P., et al. 2007, Msngr, 127, 44

Fransson, C., \& Kozma, C. 1993, ApJL, 408, L25

Fransson, C., \& Kozma, C. 2002, NewAR, 46, 487

Grebenev, S. A., Lutovinov, A. A., Tsygankov, S. S., \& Winkler, C. 2012, Natur, 490,373

Jerkstrand, A., Fransson, C., \& Kozma, C. 2011, A\&A, 530, A45

Kumagai, S., Shigeyama, T., Nomoto, K., \& Hashimoto, M. 1991, A\&A, 243, L13

Kurfess, J. D., Johnson, W. N., Kinzer, R. L., et al. 1992, ApJL, 399, L137

Larsson, J., Fransson, C., Östlin, G., et al. 2011, Natur, 474, 484

Leibundgut, B., \& Suntzeff, N. B. 2003, in Supernovae and Gamma-Ray Bursters, ed. K. Weiler (Lecture Notes in Physics, Vol. 598; Berlin: Springer), 77

Leising, M. D. 2006, ApJ, 651, 1019

Lodders, K. 2003, ApJ, 591, 1220

Lundqvist, P., Kozma, C., Sollerman, J., \& Fransson, C. 2001, A\&A, 374, 629

Magkotsios, G., Timmes, F. X., Hungerford, A. L., et al. 2010, ApJS, 191, 66

Magkotsios, G., Timmes, F. X., \& Wiescher, M. 2011, ApJ, 741, 78

Motizuki, Y., \& Kumagai, S. 2004, NewAR, 48, 69

Nagataki, S., Hashimoto, M.-A., Sato, K., \& Yamada, S. 1997, ApJ, 486, 1026

Nagataki, S., Hashimoto, M.-A., Sato, K., Yamada, S., \& Mochizuki, Y. S. 1998, ApJL, 492, L45

Nomoto, K., Tominaga, N., Umeda, H., Kobayashi, C., \& Maeda, K. 2006, NuPhA, 777, 424 
Pinto, P. A., Woosley, S. E., \& Ensman, L. M. 1988, ApJL, 331, L101

Rauscher, T., Heger, A., Hoffman, R. D., \& Woosley, S. E. 2002, ApJ, 576, 323

Röpke, F. K., Kromer, M., Seitenzahl, I. R., et al. 2012, ApJL, 750, L19

Rosman, K. J. R., \& Taylor, P. D. P. 1998, JPCRD, 27, 1275

Seitenzahl, I. R. 2011, PrPNP, 66, 329

Seitenzahl, I. R., Taubenberger, S., \& Sim, S. A. 2009, MNRAS, 400, 531

Suntzeff, N. B. 1997, arXiv:astro-ph/9707324

Suntzeff, N. B., Phillips, M. M., Elias, J. H., Walker, A. R., \& Depoy, D. L. 1992, ApJL, 384, L33

Sunyaev, R., Grebenev, S., Kaniovsky, A., et al. 1991, in AIP Conf. Proc. 232, Gamma-Ray Line Astrophysics, ed. P. Durouchoux \& N. Prantzos (Melville, NY: AIP), 211
Thielemann, F.-K., Nomoto, K., \& Hashimoto, M.-A. 1996, ApJ, 460, 408

Timmes, F. X., Woosley, S. E., Hartmann, D. H., \& Hoffman, R. D. 1996, ApJ, 464, 332

Tur, C., Heger, A., \& Austin, S. M. 2010, ApJ, 718, 357

Varani, G. F., Meikle, W. P. S., Spyromilio, J., \& Allen, D. A. 1990, MNRAS, 245,570

Wang, L., Wheeler, J. C., Kirshner, R. P., et al. 1996, ApJ, 466, 998

Wongwathanarat, A., Janka, H.-T., \& Müller, E. 2010, ApJL, 725, L106

Woosley, S. E., Hartmann, D., \& Pinto, P. A. 1989, ApJ, 346, 395

Woosley, S. E., \& Hoffman, R. D. 1991, ApJL, 368, L31

Woosley, S. E., \& Weaver, T. A. 1995, ApJS, 101, 181 